\title{
Effects of knowledge of results on mixed schema discrimination
}

ED M. EDMONDS AND MARVIN R. MUELLER, AUGUSTA COLLEGE SELBY H. EVANS, TEXAS CHRISTIAN UNIVERSITY

In a mixed schema task, Ss learned to distinguish among different schemata both with and without knowledge of $r e-$ sults (KR). KR did not appear to assist schema learning. These results indicate that humans can discriminate higher order variables (schemata) without external assistance.

Schema theory has suggested (see Attneave, 1957) that recurrent regularities in the environment may be encoded into schemata as a means of reducing information requirements in memory tasks. Attneave (1957) showed that familiarity with a prototype of stimuli used in a paired-associates task facilitated performance. The natural environment, however, seems to present a much more difficult problem; there may be no prototype and members of different schema families may be mixed together. Edmonds \& Evans (1966) have shown that Ss can abstract a schema from a set of varying instances without the aid of a prototype. Evans \& Edmonds (1966) have shown that with knowledge of results (KR) Ss can readily learn to distinguish patterns of one schema family from random patterns (having no schema), and also from patterns of another schema family.

The present studies represent two more steps toward demonstrating schema learning under conditions which may more closely approximate those of the natural environment. The environment cannot be assumed to provide training on a single schema, or even a pair of schemata, at one time. There is, perhaps, a schema associated with resting butterflies; another, with resting grasshoppers; another, with resting moths; another, with resting wasps. From time to time, instances of these and many other schemata are available, but to abstract several schemata from a mixture of such instances would require the ability to recognize new instances of a particular schema while learning the characteristics of the schema. The information required to assign instances to the correct schema family might be provided by a more knowledgeable source in the form of $\mathrm{KR}$ or labeling. On the other hand, schema learning would be much more efficient if it did not depend on external reinforcement for assigning instances to the appropriate schema family. It has been shown (Evans, 1964) that when substantial constraint redundancy is present, both human Ss and a computer program can learn to separate patterns from two different schema families using only the information in the patterns themselves (i.e., without KR).

These considerations raise two questions which are dealt with in Experiments 1 and 2. First, can Ss readily learn to distinguish among different schemata when the task includes several different schemata? Second, can Ss learn to distinguish among these schemata without external assistance such as is provided by KR? Experiment 1

Subjects. The Ss were 33 undergraduates enrolled in psychology courses at Texas Christian University, and they were naive with respect to the type of patterns used in this study.

Patterns. The patterns were produced by a computer program, VARGUS 7 (Edmonds \& Evans, 1966; Evans, 1964), which draws histoform patterns at random from a defined population and allows independent manipulation of schema and redundancy. The schema in patterns produced by VARGUS 7 is composed of the most probable sequence (MPS) of column heights as determined by the transitional probabilities of a seven element Markov process. In the present experiment, each of four different most probable column height sequences (MPSs) was used with VARGUS 7 to produce $67 \%$ redundant patterns.

Task and procedure. The Ss were given a 15 page booklet with three patterns printed on each page and they were instructed to select the pattern on each page that "least resembled" the other two patterns. All three patterns on a page were different. Two of the patterns on a page, however, were chosen at random from one of four MPSs while the third pattern was chosen at random from one of the three remaining MPSs. The pairing of MPSs thus varied randomly from page to page. The positions of the three patterns on each page were randomly assigned. The Ss were allowed $45 \mathrm{sec}$. to choose a pattern on each page and write their response. They were then told which pattern was correct. Experiment 2

All aspects (including the pattern booklets) of this experiment were identical with Experiment 1, except that a new group of $33 \mathrm{Ss}$ was used and no $\mathrm{KR}$ was provided.

\section{Results and Discussion}

The Ss in Experiment 1 will be referred to as KR Ss and those in Experiment 2 will be referred to as NKR Ss.

In Fig. 1 the mean number of correct pattern selections for KR Ss and NKR Ss are plotted as a function of blocks of three trials. Comparisons based on trial block 5 showed that both groups performed significantly $(t=3.18, d f=32, p<.01$, for KR Ss) more accurately than chance.

Figure 1 shows that the performance level on block 5 was approximately the same for both groups, but earlier 


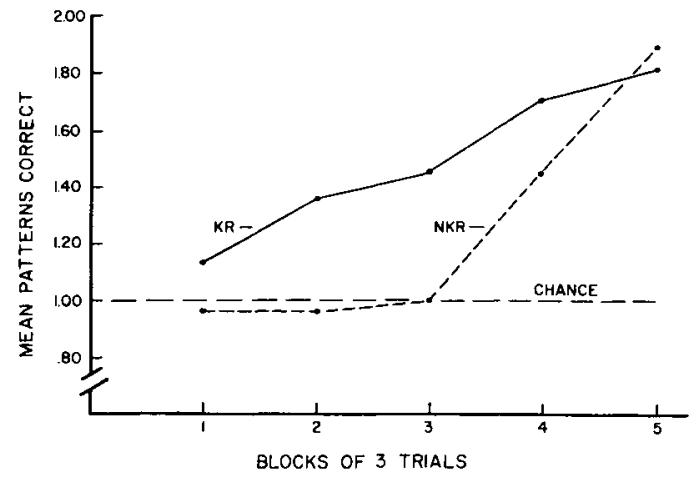

Fig. 1. Block means for KR Ss and NKR Ss.

portions of the curves are quite different. The NKR Ss initially performed no better than chance and then showed a sudden and rapid rise to a high performance level. The performance of the KR Ss increased almost linearly over the series of trials.

The results with the KR Ss are quite similar to previous results (Evans \& Edmonds, 1966) in a similar task involving only two schemata. The mixing of four schemata together does not seem to have made the task appreciably more difficult. Evidently, humans can readily learn to discriminate among several schemata at once. The results with the NKR Ss demonstrate that $K R$ is not needed to achieve discrimination among schemata in the oddity task. The information in the patterns themselves is evidently sufficient to allow selection of the relevant variables. These results are in accord with a previous study (Evans, 1964) using a sorting task.

The curve for the NKR Ss is strikingly different from the curve obtained with $\mathrm{KR}$ in this task. The steady performance at chance level, followed by a rapid and consistent rise to a high level of performance, suggests the term insight. At any rate, the processes governing improvement seem to be quite different in the two cases. This curve must be interpreted with caution, however, because the same sequence of patterns was presented to all Ss. Thus, one of the sets of patterns in block three or four may have had some characteristic which provided a strong clue as to how the oddity instructions should be interpreted. On the other hand, a corresponding effect is not present with the $\mathrm{KR} \mathrm{Ss}$, who received the same sequence of patterns.

In the present task, there are really two things to be learned or discovered: first, the relevant dimensions along which oddity is to be sought, and second, the particular characteristics of each schema. The sche- mata could be learned without affecting performance if Ss did not also recognize that the schemata constituted the relevant dimensions. Thus, the NKR Ss may have been learning the schemata in the first nine trials even though their performance did not improve until they also "recognized" that each set of patterns had two patterns of the same schema and one of a different schema. (The term "recognized"' is used with reservation here because post-experiment questioning did not indicate that Ss were aware of the principle to which they were responding.) Indeed the effect of $\mathrm{KR}$ in this task may be primarily to indicate the relevant variable rather than to assist schema learning. This speculation is suggested by the similar performance of the KR and NKR Ss in the last block of trials.

The present results offer additional support for the view that schema learning should play an important role in a theory of perceptual learning. In accord with Gibsons" (1955) formulation, Ss can learn to discriminate these higher order variables without $\mathrm{KR}$. The question of learning without reinforcement is not involved here. Finding an orderly pattern in figures which initially seemed chaotic may indeed be reinforcing; anecdotal evidence supports that view. Finding a schema also reduces the technical information or uncertainty of a set of patterns, and such reduction might be reinforcing in Berlyne's (1960) theory. The important point is that Ss found the schemata without external assistance.

If spontaneous schema learning is a general process, humans (and perhaps other animals) may be able to do a great deal of schema learning on their own. Perhaps one of the functions of curiosity behavior is to afford opportunity for schema learning. At any rate, the opportunities for schema learning are vastly increased if it can be done with only the information in the patterns themselves.

\section{References}

Attneave, F. Transfer of experience with a class-schema to identification-learning of patterns and shapes. J. exp. Psychol., 1957, $54,81-88$.

Berlyne, D. E. Conflict, arousal, and curiosity. New York: McGrawHill, 1960.

Edmonds, E. M., \& Evans, S. M. Schema learning without a prototype. Psychon. Sci., 1966, 5, 247-248.

Evans, S. H. A model for perceptual category formation. Unpublished doctoral dissertation. Texas Christian University, 1964.

Evans, S. H., \& Edmonds, E. M. Schema discrimination as a function of training. Psychon. Sci., 1966, 5, 247-248.

Gibson, J. J., \& Gibson, E. J. Perceptual learning: differentiation or enrishment? Psychol. Rev., 1955, 62, 32-41. 